\title{
Insights from Spitzer on massive star formation in the LMC
}

\author{
Rémy Indebetouw ${ }^{1}$, Barbara A. Whitney ${ }^{2}$, Marta Sewilo ${ }^{3}$, Thomas \\ Robitaille $^{4}$, Margaret Meixner ${ }^{3}$ and the (rest of the) SAGE team \\ ${ }^{1}$ University of Virginia and National Radio Astronomy Observatory, P.O. Box 400325, \\ Charlottesville, VA, 22904 USA, email: remy@virginia.edu \\ ${ }^{2}$ Space Science Institute, Boulder, CO, USA \\ ${ }^{3}$ Space Telescope Science Institute, Baltimore, MD, USA \\ ${ }^{4}$ Smithsonian Astrophysical Observatory, Cambridge, MA, USA
}

\begin{abstract}
Spitzer's sensitive mid-IR photometric surveys of the Magellanic Clouds provide a relatively extinction-free census of star formation activity, and sub-parsec resolution permits the study of individual massive protostars and small clusters. Using the SAGE survey of the LMC, we identify over 1000 massive YSO candidates by their MIR colors. Analysis of their spectral energy distributions (SEDs) constrains the stellar content and evolutionary state, beginning to realize for the first time the unique potential of the Clouds to study an entire galaxy's population of individual protostars. We probe the physics underlying the Schmidt-Kennicutt scaling law by analyzing how it begins to break down at $10-100 \mathrm{pc}$ spatial scales. MIR spectroscopic surveys currently underway like SAGE-SPEC will enable us to couple the circumprotostellar dust distribution (the evolutionary state reflected in the SED) with the physical state of the gas, dust and ice.
\end{abstract}

Keywords. surveys, stars: formation, ISM: clouds, H II regions, galaxies: individual (LMC), Magellanic Clouds

\section{Introduction}

Surveying the Agents of a Galaxy's Evolution (SAGE, Meixner et al. 2006)) is a Spitzer Space Telescope legacy program to image the entire Large Magellanic Cloud (LMC) with the IRAC (3.6, 4.5, 5.8, \& $8.0 \mu \mathrm{m}$, Fazio et al. 2004) and MIPS (24, 70, \& $160 \mu \mathrm{m}$, Rieke et al. 2004) cameras. SAGE provides a complete census of stars and the interstellar medium in the entire galaxy down to the confusion limit, except in bright H II regions where fainter point sources are lost in bright diffuse emission. SAGE can study the life cycle of dust - formation, processing, and destruction - in the ISM down to column densities of $1.2 \times 10^{21} \mathrm{~cm}^{-2}$. SAGE can also find and characterize all massive young stellar objects in the entire galaxy. The SAGE LMC survey has been followed up by a large-scale photometric survey of the Small Magellanic Cloud and part of the Bridge (SAGE-SMC; PI Gordon), and several mid-infrared spectroscopic surveys. These include a complete spectral cube of 30 Doradus (Indebetouw et al. 2009), spectra of 200 Young Stellar Object (YSO) candidates (PI Looney), and of several hundred young and evolved stars (SAGE-SPEC, PI Tielens).

These mid-infrared observations are greatly improving our understanding of star formation, massive (proto)stellar feedback, and physical conditions in the ISM of the Magellanic system. In particular, we can address aspects of star formation that take place on meso-scales, between 0.1 and $1000 \mathrm{pc}$. The very small scales of star formation are best studied in our own Galaxy, and for low-mass (solar or less) stars, these detailed nearby 
studies have resulted in a fairly complete theory. Individual high-mass star formation still holds many puzzles at high spatial resolution, but if we assume that there is consistency in how a cluster of stars forms from a molecular clump (parsec-scale), then we can use the SAGE data to connect those individual small clusters and clumps to the kiloparsec and larger-scale star formation, scaling relations commonly used for galaxies as a whole. We can in particular address:

- physical conditions in clouds and cores;

- stability/collapse/triggering of clouds;

- distribution of SF regions in space/time;

- feedback from protostars on clouds.

\section{YSO candidates: selection and population analysis}

Figure 1 shows one of the many color-magnitude diagrams that can be constructed using SAGE point source photometry. Various known populations are marked. YSOs occupy the bright red part of mid-IR color-magnitude space. Main sequence stars have nearly zero infrared color, evolved stars have modestly red colors except for a relatively rarer population of extreme AGB stars and proto-planetary nebula which can be bright and quite red. Thus a careful set of color-magnitude cuts can isolate YSO candidates reasonably reliably (spectroscopic confirmation is required for certainty of course). As described in detail in Whitney et al. (2008), we can select over 1000 massive YSO candidates, greatly increasing the statistical sample relative to the $\sim$ dozen massive YSOs in the LMC previously described in the literature. At fainter magnitudes, reddened and redshifted background galaxies begin to contaminate any sample, effectively limiting this analysis to greater than $5-10 \mathrm{M}_{\odot}$ (SAGE sensitivity provides photometry of isolated protostars down to $\sim 3 \mathrm{M}_{\odot}$ depending on evolutionary state).

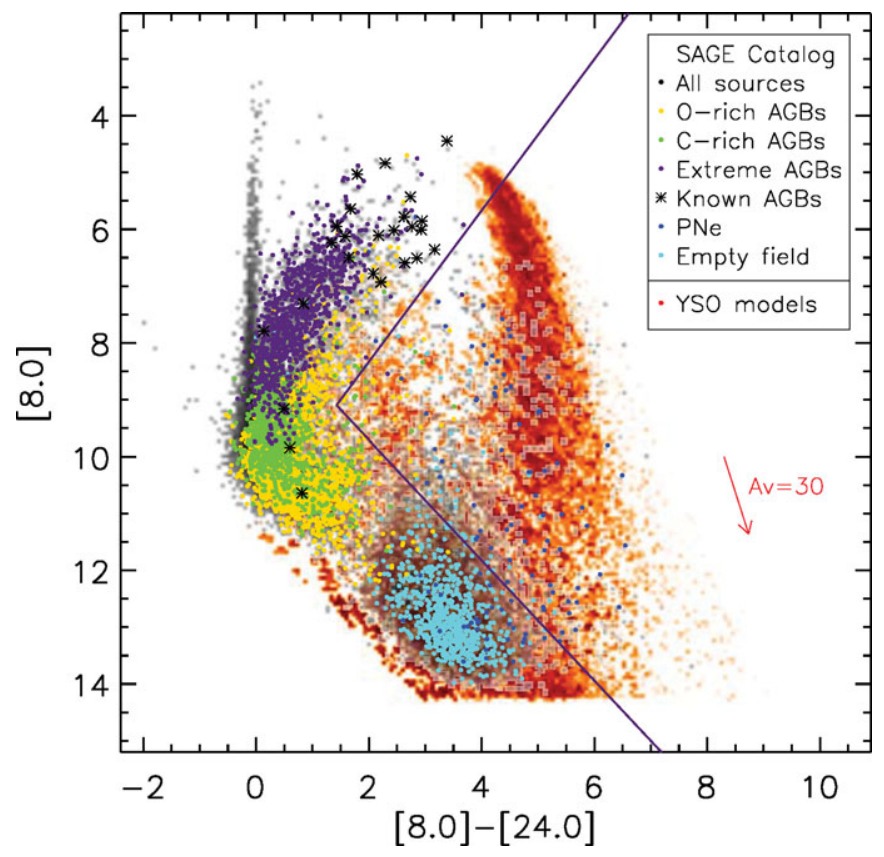

Figure 1. A SAGE color-magnitude diagram showing the locations of known object populations, modeled young stellar objects (YSOs), and a selection cut used to isolate YSO candidates. 


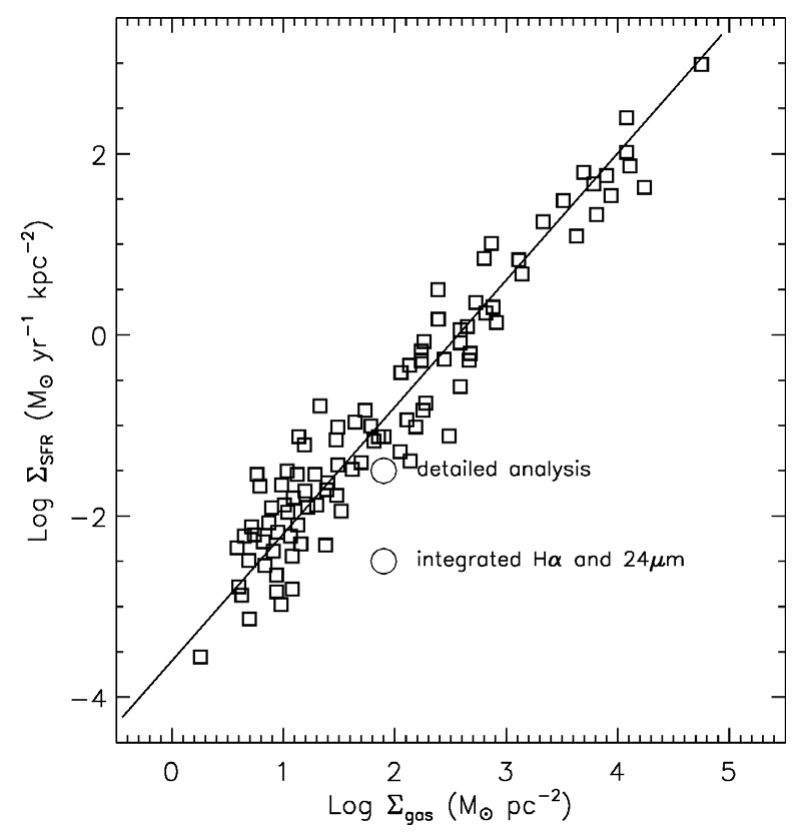

Figure 2. The molecular ridge south of 30 Doradus falls below the Schmidt-Kennicutt relation (Kennicutt 1998) in total emission because each of its regions is a poor cluster, not fully sampling the hot end of the stellar initial mass function. When the individual regions are analyzed, their mass does sum to something consistent with SK.

The YSO catalog permits many different analyses. We find that the YSOs are highly correlated with the gas surface density and are relatively strongly clustered with each other (Whitney et al. 2008). The spectral energy distribution of a YSO can be compared against a grid of dust radiative transfer models (Robitaille et al. 2007), constraining the central mass and luminosity (spectral class given a set of pre-main-sequence evolutionary tracks), and the circumstellar dust distribution (disk vs. envelope) and total mass (corresponding to a relative evolutionary state from younger strongly accreting sources to older sources with remnant disks). We can also analyze the stability of individual molecular clouds and clumps; for example in the molecular ridge south of 30 Doradus we find that the clouds with a higher ratio of CO luminosity (or molecular gas mass assuming a constant X-factor conversion) to virial mass have greater $24 \mu \mathrm{m}$ and total infrared luminosities. Considering the entire galaxy, Yang et al. (2007) find that the YSO candidates are found in Toomre-unstable regions, but only if one includes the gravitational potential of the stars as well as the gas in the instability criterion.

We can use SED analysis of YSOs and small HII regions to dissect the SchmidtKennicutt (SK) scaling relation at unprecedented small scales (few tens of parsecs). The relation states that star formation rate (measured by infrared plus $\mathrm{H} \alpha$ or UV emission, Calzetti et al. 2005; Kennicutt et al. 2007) is proportional to the gas surface density to the 1.4 power. It is commonly used in extragalactic work and holds tightly above kiloparsec scales across Hubble type. However, we expect it to break down around the Giant Molecular Cloud scale (100 pc or so), where local conditions may not be sufficiently averaged. In Indebetouw et al. (2008) we analyze the strangely faint southern molecular ridge of the LMC, south of 30 Doradus. The total $24 \mu \mathrm{m}$ and $\mathrm{H} \alpha$ emission from the ridge places it below the SK relation (Figure 2), but if one analyzes each region individually, one finds that they are mostly poor clusters that are not fully sampling the initial mass 


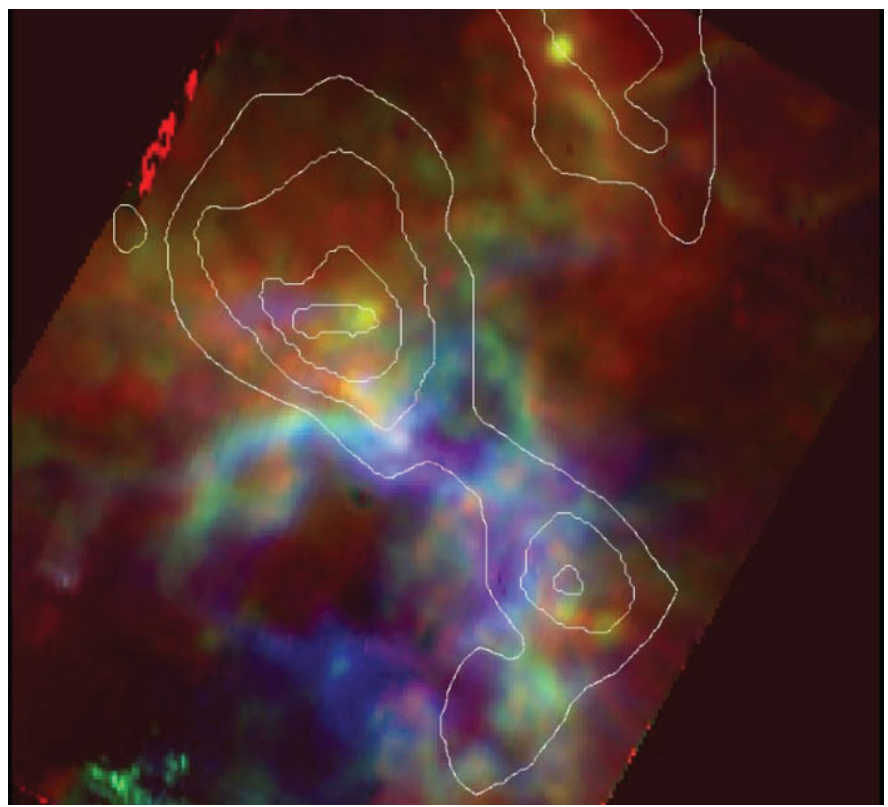

Figure 3. 30 Doradus in the mid-infrared showing the ionization structure of the bubble $\mathrm{H}$ II region. blue $=[\mathrm{NeIII}]$, green $=[\mathrm{NeII}]$, red $=8 \mu \mathrm{m}$ PAH feature (continuum subtracted) contours $={ }^{12} \mathrm{CO}(1-0)$ (Johansson et al. 2008).

function (IMF). With a few 1000 stars and most massive stars only of B type, they do not have the total luminosity that one would expect for a fully sampled IMF. Their mass does add up to a total consistent with SK, but the mode of star formation is non-typical and doesn't include rich bright clusters.

\section{Spectroscopy and feedback}

Mid-infrared spectra contain several pairs of atomic fine-structure lines which can be used to constrain the ionization parameter and ionizing field in an H II region (e.g., Morisset et al. 2004). We apply this analysis to 30 Doradus, shown in Figure 3 . The ionization gradient from inside the bubble out into the molecular cloud is already clear from the transition from [Ne III] (blue) to [Ne II] (green). Photoionization modeling reveals a similar structure, of a hot bubble ionized by a relatively hard radiation field (especially a the eastern end which is dominated by a Wolf-Rayet star). Local features from individual hot stars and the remnant molecular material are much stronger than any radial gradient from R 136 (Indebetouw et al. 2009).

As described by K. Sandstrom at this meeting, Spitzer spectra also contain aromatic dust emission features (usually attributed to polycyclic aromatic hydrocarbons, PAHs) which allow one to determine the ionization state of small dust in photo-dissociation regions around YSOs and HII regions, and to study the destruction of dust by massive (proto)stars. As described by J. Oliveira and T. Shimonishi (elsewhere in this volume), we can also make detailed analyses of the circumstellar dust and ices around protostars, leading to much finer constraints on their evolutionary state and the early stages of the heating and processing of their natal material.

In conclusion, we have only just begun to learn from the massive mid-infrared datasets of the Magellanic system provided by the Spitzer Space Telescope, especially the SAGE, 
SAGE-SMC, and SAGE-SPEC surveys. We have already increased the number of known massive protostellar objects by several orders of magnitude in the LMC, characterized their population and evolutionary state, determined stability conditions locally and globally, and will continue to examine the physics behind extragalactic star formation scaling laws. Spectra of H II regions and YSOs in the Clouds will yield even more detail on their physical conditions, and the formation and feedback of massive stars as a function of metallicity and galactic environment.

\section{References}

Calzetti, D., Kennicutt, R. C., Bianchi, L., et al. 2005, ApJS, 633, 871

Fazio, G. G., Hora, J. L., Allen, L. E., et al. 2004, ApJS, 154, 10

Indebetouw, R., De Messieres, G., et al. 2009, AJ, submitted

Indebetouw, R., Whitney, B. A., Kawamura, A., et al. 2008, AJ, 136, 1442

Johansson, L. E. B., Greve, A., Booth, R. S., et al. 1998, A\&A, 331, 857

Kennicutt, R. C., Jr. 1998, ApJ, 498, 541

Kennicutt, R. C., Jr., Calzetti, D., Walter, F., et al. 2007, ApJ, 671, 333

Meixner, M., Gordon, K. D., Indebetouw, R., et al. 2006, AJ, 132, 2268

Morisset, C., Schaerer, D., Bouret, J.-C., \& Martins, F. 2004, A\& A, 415, 577

Rieke, G. H., Young E. T., Engelbracht C. W., et al. 2004, ApJS, 154, 25

Robitaille, T. P., Whitney, B. A., Indebetouw, R., \& Wood, K. 2007, ApJS, 169, 328

Whitney, B. A., Sewilo, M., Indebetouw, R., et al. 2008, AJ, 136, 18

Yang, C.-C., Gruendl, R. A., Chu, Y.-H., Mac Low, M.-M., \& Fukui, Y. 2007, ApJ, 671, 374 